\title{
Análisis estructural de los problemas orientado al aprendizaje de Operaciones de Separación
}

\section{A. Santafé-Moros ${ }^{a}$, J.M. Gozálvez-Zafrillab, J. Navarro Laboulais ${ }^{c}$}

EICE ASEI (Aplicación de la simulación en la enseñanza de la Ingeniería). Departamento de Ingeniería Química y Nuclear. ETSII. Universitat Politècnica de València. aassanmo@iqn.upv.es, bjmgz@iqn.upv.es, cjnavarla@iqn.upv.es

\begin{abstract}
The subject Unit Operations of the Bachelor degree in Chemical Engineering is focused on learning the common operations used in chemical processes. After our experience, most of the students perceive that the subject is made up of independent learning units which requires the study of a great amount of theoretical concepts involving several unconnected calculations for each one, so they think the subject is tedious and complex. The reason is that they are not aware about the similarities among the unit operations. Besides, the procedures required to solve full exercises are hard for them and they misunderstood the reasoning process. A deep analysis of the exercises used to learning the unit operation calculations was carried out. Besides, the main features of mathematical software used (Mathcad) were considered. The aim is to do the reasoning process more visual, to facilitate the awareness of the transversality of the several unit operations and to reduce the time spent on the problem-solving. The result has been the restructuration of the exercise by means of "calculation areas" and the implementation of "function libraries", which has improved the learning outcomes.
\end{abstract}

Keywords: transversality, integration, problem-solving, unit operations, Mathcad

\begin{abstract}
Resumen
La asignatura Operaciones de Separación del Grado de Ingeniería Química se centra en el aprendizaje de las operaciones de mayor aplicación en procesos de ingeniería química. La falta de conciencia de las similitudes entre las distintas operaciones hace que una gran parte de los alumnos tenga la visión de que la asignatura está formada por unidades inconexas que requieren el estudio de muchos conceptos teóricos con cálculos diferenciados para todos ellos, de manera que les parece poco atractiva,
\end{abstract}


Análisis estructural de los problemas orientado al aprendizaje de Operaciones de Separación

compleja y voluminosa. La resolución de un problema completo les resulta, en general, larga y tienden a perderse en el proceso de razonamiento. Estas dificultades se han hecho más evidentes en los estudios de Grado, respecto a la misma asignatura de la anterior titulación de Ingeniero Químico. Por ello, se ha realizado un profundo análisis de los problemas utilizados para aprender los cálculos de cada operación, así como de las características del software utilizado en su resolución (Mathcad). Todo ello con el objetivo de hacer facilitar el proceso de razonamiento y la conciencia de transversalidad entre las distintas operaciones y de disminuir el tiempo requerido. Como resultado se han restructurado los problemas con el uso de "zonas de cálculo" y la creación de "librerías de funciones", lo que ha supuesto una mejora en el aprendizaje.

Palabras clave: Transversalidad, integración, resolución de problemas, Operaciones de Separación, Mathcad

\section{Introducción}

Este trabajo está enmarcado dentro del PIME "Desarrollo de estrategias para la mejora del aprendizaje y evaluación de los problemas de Operaciones de Separación” desarrollado por miembros del EICE ASEI.

La asignatura Operaciones de Separación del grado de Ingeniería Química se centra en el aprendizaje de las operaciones unitarias de transferencia de materia de mayor aplicación en procesos de ingeniería química. Para cada una de las operaciones estudiadas el alumno debe aprender los fundamentos físicos, las características y los cálculos para el diseño de los equipos. Si bien cada operación tiene un fundamento propio, todas tienen como base la transferencia de materia, requieren operar con datos de equilibrio y de propiedades físicas, y la realización de balances de materia y en su caso de energía.

A nivel de grado estas operaciones se limitan al tratamiento de sistemas de dos o tres componentes para los que la mayor parte de los métodos de cálculo se basan en procedimientos gráficos. De esta manera, tradicionalmente en muchas escuelas, la resolución de los ejercicios se viene realizando mediante el uso de calculadora y papel milimetrado. Sin embargo, el uso de un programa matemático permite realizar problemas más complejos que emplean cálculos iterativos y facilitar el estudio del efecto de las variables del proceso. Por ello, desde la aparición de la titulación de Ingeniero Químico, en la ETSII, se optó por utilizar el programa Mathcad en la resolución de problemas debido a su entorno amigable y de fácil comprensión para el alumno. Otros docentes (Harb, 1997; Sánchez Mirón) también han apostado por este software en la enseñanza de la Ingeniería 
Química. Esto llevó a organizar la asignatura en sesiones en las que se desarrollan los conceptos teóricos y los métodos de cálculo y en sesiones de aula informática para la resolución de un problema de una operación concreta (Santafé, 2013).

A pesar de que los profesores relacionan las distintas operaciones, una gran parte de los alumnos no es consciente de las similitudes entre las distintas operaciones lo que hace que tengan una visión de que la asignatura está formada por unidades de aprendizaje inconexas que requieren el estudio de muchos conceptos teóricos con cálculos diferenciados para todos ellos, de manera que la asignatura les resulta poco atractiva, compleja y voluminosa.

Por ello, el aprendizaje de estas operaciones resultará más fácil si el alumno adquiere conciencia de las similitudes entre las operaciones y de que el aprendizaje de cada una de ellas sirve de punto de partida para el estudio de las siguientes.

Ante esta evidencia, y para transmitir esta visión al alumno, se realizó un análisis que dio como resultado una estructuración similar de las sesiones de teoría y la estructuración de la resolución de problemas en pasos similares y unidades intercambiables.

\section{Objetivos}

En este trabajo nos planteamos realizar un análisis estructural de los problemas de la materia operaciones de separación en ingeniería química.

Mediante este análisis pretendemos identificar procedimientos de razonamiento y de cálculo comunes entre los diferentes tipos de problemas, todo ello con el objetivo general de establecer una metodología de resolución de problemas, apoyada con software matemático, que facilite el aprendizaje del alumno.

Para la consecución de este objetivo global se han establecido los siguientes objetivos particulares:

- Analizar los problemas para identificar los procesos de razonamiento y las estructuras de cálculo comunes en la resolución de los problemas de los distintos tipos de operaciones de separación.

- Desarrollar una metodología de resolución basada en la utilización del software matemático adecuada a la estructuración realizada.

- Transmitir al alumno estrategias de realización de los problemas de manera que sea capaz de identificar la similitud entre los razonamientos y las estructuras de cálculo a efectuar en las distintas operaciones, así como de aplicarlas para resolver situaciones nuevas.

- Trasladar la estructuración al planteamiento y la evaluación del examen.

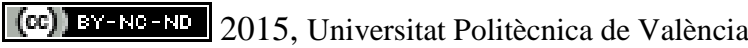


Análisis estructural de los problemas orientado al aprendizaje de Operaciones de Separación

\section{Desarrollo de la innovación}

El trabajo requirió el análisis por parte de los profesores de los problemas tipo trabajados en el aula para el aprendizaje de las operaciones de separación que se tratan en la asignatura.

A partir del análisis anterior, se hizo un estudio de las características del software que permitiera adaptar su uso a una forma más estructurada de realizar los problemas.

Todo el análisis sirvió para reorganizar los ficheros de resolución de los ejercicios de forma que facilitará la identificación de la similitud entre las distintas operaciones.

En la fase final se trasladó la metodología de estructuración de los problemas al planteamiento y la resolución de los ejercicios de examen.

En este primer año de introducción de la metodología, la evaluación de la consecución de los objetivos se centró en verificar si los alumnos han captado la similitud entre los procedimientos de cálculo. Para ello se realizó una encuesta en la que se analizó la percepción que tienen los alumnos de la asignatura en general y de la metodología empleada en particular.

\section{Resultados}

A continuación vamos a ilustrar con ejemplos el proceso realizado. Para ello es necesario primero contextualizar la asignatura. A nivel de Grado se estudian los cálculos de las operaciones más comunes en la industria química: absorción, destilación, extracción líquido-líquido, extracción sólido-líquido y evaporación. Algunas de estas operaciones pueden aplicarse en un proceso de una etapa o varias etapas conectadas en flujo cruzado, como son la destilación instantánea (FLASH) y la diferencial (DIF), la extracción líquidolíquido (ELL) y sólido-líquido (ESL) y la evaporación (EVAP). Otras se operan a nivel industrial en procesos de varias etapas a contracorriente, éstas son la absorción (ABS), la rectificación (MT para método de McCabe-Thiele; PS para método Ponchon-Savarit) y la extracción líquido-líquido.

Como resultado del análisis de los problemas se identificaron los procedimientos de cálculo o de razonamiento que deben realizarse para diseñar las distintas operaciones. La Tabla 1 muestra la matriz de relación entre las operaciones estudiadas y los tipos de cálculos requeridos en su resolución. A su vez, cada tipo de cálculo se dividió en los cálculos específicos necesarios para llegar a su consecución. En este nivel de subdivisión de los problemas se identificó de qué asignatura provenían los conocimientos previos y las habilidades requeridas para su resolución. 
A. Santafé-Moros, J.M. Gozálvez-Zafrilla, Javier Navarro Laboulais

Tabla 1. Matriz de relación entre las operaciones y los tipos de cálculos requeridos

\begin{tabular}{|c|c|c|c|c|c|c|c|c|c|}
\hline & \multicolumn{4}{|c|}{$\begin{array}{l}\text { Operaciones de etapas a } \\
\text { contracorriente }\end{array}$} & \multicolumn{5}{|c|}{ Operaciones en una etapa } \\
\hline & ABS & MT & PS & ELL & FLASH & DIF & ELL & ESL & EVAP \\
\hline Geometría & $\mathrm{x}$ & $\mathrm{x}$ & $\mathrm{x}$ & $\mathrm{x}$ & $\mathrm{x}$ & & $\mathrm{x}$ & $\mathrm{x}$ & \\
\hline Reparto & & & $\mathrm{x}$ & $\mathrm{x}$ & $\mathrm{x}$ & & $\mathrm{x}$ & $\mathrm{x}$ & \\
\hline $\begin{array}{l}\text { Equilibrio modelo } \\
\text { termodinámico }\end{array}$ & $\mathrm{x}$ & & & & & & & & \\
\hline Datos de equilibrio & $\mathrm{x}$ & $\mathrm{x}$ & $\mathrm{x}$ & $\mathrm{x}$ & $\mathrm{x}$ & $\mathrm{x}$ & $\mathrm{x}$ & $\mathrm{x}$ & \\
\hline $\begin{array}{l}\text { Datos de propiedades } \\
\text { físicas }\end{array}$ & & $\mathrm{x}$ & $\mathrm{x}$ & $\mathrm{x}$ & $\mathrm{x}$ & $\mathrm{x}$ & $\mathrm{x}$ & $\mathrm{x}$ & $\mathrm{x}$ \\
\hline $\begin{array}{l}\text { Balances en corrientes } \\
\text { entrantes y salientes }\end{array}$ & $\mathrm{x}$ & $\mathrm{x}$ & $\mathrm{x}$ & $\mathrm{x}$ & $\mathrm{x}$ & $\mathrm{x}$ & $\mathrm{x}$ & $\mathrm{x}$ & $\mathrm{x}$ \\
\hline Condición mínima & $\mathrm{x}$ & $\mathrm{x}$ & $\mathrm{x}$ & $\mathrm{x}$ & & & & $\mathrm{x}$ & \\
\hline Línea o polo de operación & $\mathrm{x}$ & $\mathrm{x}$ & $\mathrm{x}$ & $\mathrm{x}$ & & & & & \\
\hline $\begin{array}{l}\text { Identificación del punto de } \\
\text { partida del cálculo de } \\
\text { etapas }\end{array}$ & $\mathrm{x}$ & $\mathrm{x}$ & $\mathrm{x}$ & $\mathrm{x}$ & & & & & \\
\hline $\begin{array}{l}\text { Programa del cálculo de } \\
\text { etapas }\end{array}$ & $\mathrm{x}$ & $\mathrm{x}$ & $\mathrm{x}$ & $\mathrm{x}$ & & & & & \\
\hline Representación gráfica & $\mathrm{x}$ & $\mathrm{x}$ & $\mathrm{x}$ & $\mathrm{x}$ & $\mathrm{x}$ & & $\mathrm{x}$ & & \\
\hline Determinación de calores & & & $\mathrm{x}$ & & $\mathrm{x}$ & & & & $\mathrm{x}$ \\
\hline $\mathrm{N}^{\circ}$ mínimo de etapas & & $\mathrm{x}$ & $\mathrm{x}$ & & & & & & \\
\hline
\end{tabular}

El análisis se extendió para identificar qué aspectos específicos se introducen durante la resolución de alguna operación que puede ser extrapolable a otras. Esto además de mejorar el proceso de aprendizaje del alumno servirá como herramienta de medición del grado de comprensión en cuanto a transversalidad e integración de conceptos. Para ello en el examen, se pide a los alumnos que los cálculos practicados en el aula para una operación los adapten al cálculo de otra.

En la siguiente fase, se analizó qué partes del cálculo convenía proporcionar a los alumnos, especialmente en base a conocimientos ya trabajados en asignaturas previas, ya que al llevar tiempo su desarrollo contribuyen a que al alumno se le hagan largos los problemas, a que se pierdan en el proceso general de razonamiento del problema y en la aplicación de los conocimientos propios de la asignatura.

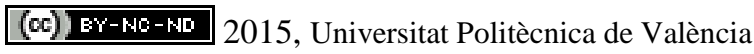

Congreso In-Red (2015) 
Como se ha comentado con anterioridad, algunos cálculos están basados en procedimientos gráficos que hay que trasladar a su equivalente matemático. Un ejemplo es lo que hemos denominado la función "reparto". La Figura 1 muestra la diapositiva utilizada en la sesión de teoría que resume el método de cálculo aplicado a la operación de destilación instantánea. Gráficamente la resolución consiste en buscar la línea que pasa por un punto de coordenadas conocidas $\left(\mathrm{z}_{\mathrm{F}}\right.$ y $\left.\mathrm{h}_{\mathrm{F}}+\mathrm{Q} / \mathrm{F}\right)$ y que tiene los extremos en la intersección con las curvas de entalpía de vapor y de líquido saturado para los que las abscisas están relacionadas por una ecuación $(\mathrm{y}=\mathrm{yEQ}(\mathrm{x}))$. Matemáticamente se puede expresar como una igualdad de las pendientes de las líneas o equivalentemente como la ecuación definida para evitar la indeterminación que puede surgir al aplicar un método numérico para obtener el valor de $\mathrm{x}$. El método de resolución se basa en el conocimiento de este tipo de diagramas explicado en la asignatura "Termodinámica química” y en los fundamentos matemáticos y de métodos numéricos de la asignatura "Métodos de cálculo en IQ".

\section{Destilación instantánea}

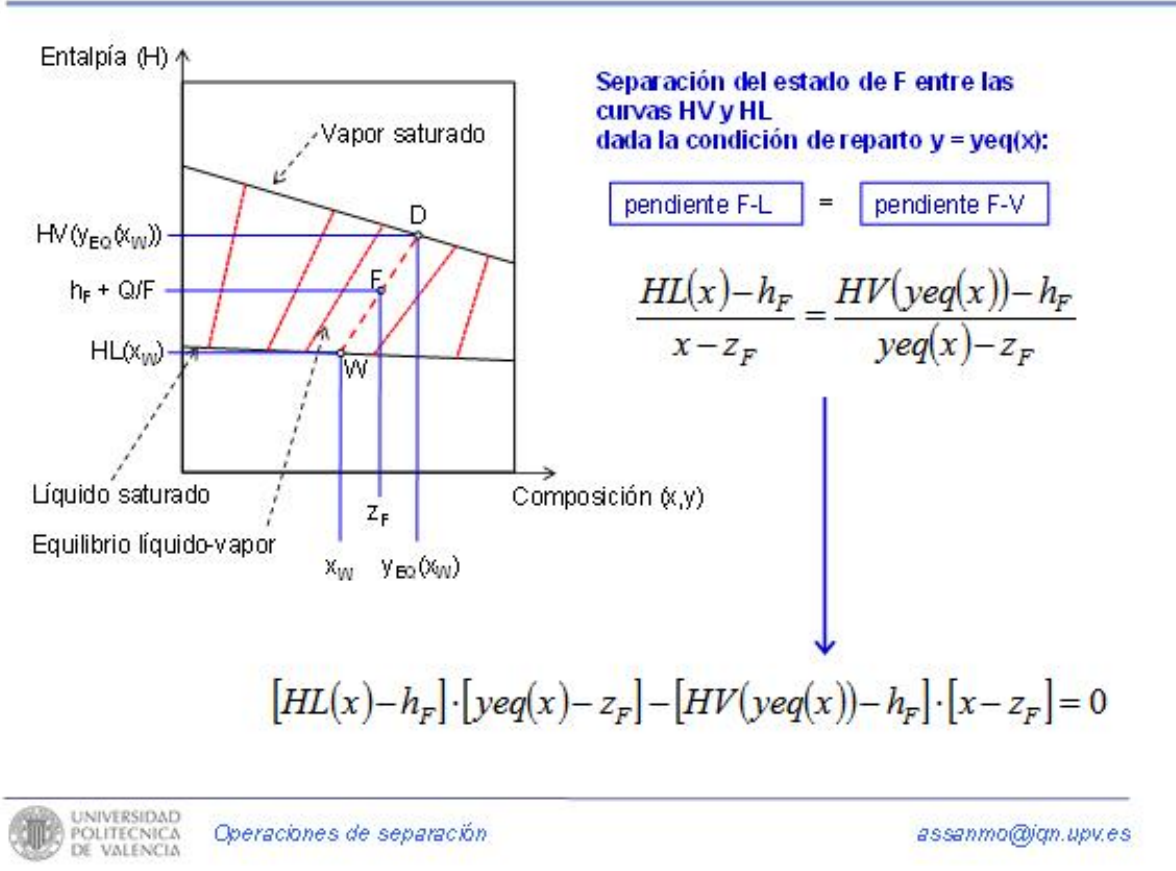

Fig. 1. Diapositiva de explicación del cálculo de un proceso de destilación instantánea 
Como se puede observar en la Tabla 1 este tipo de cálculo (reparto) se aplica en varias operaciones, para las que cambia el fundamento físico pero se mantiene el concepto geométrico y matemático. En este caso, los profesores hemos optado por crear la función de cálculo como se muestra en la Figura 2 y explicar los cálculos que hace.

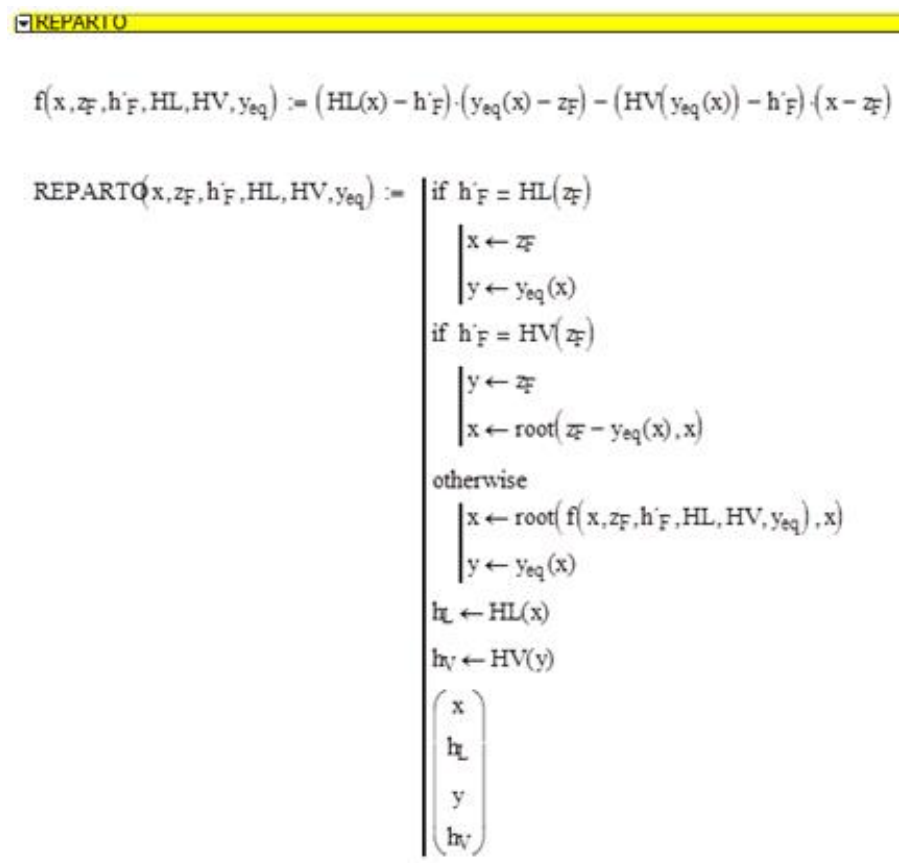

Fig. 2. Función para el cálculo del reparto de fases.

De esta manera, el alumno debe identificar cuando se puede aplicar esta función para realizar el cálculo y saber aplicarla. La Figura 3 muestra cómo se aplicaría en un cálculo concreto. También se puede observar en esta figura la aplicación de la función “y_pp”, que no es más que la ecuación de una recta que pasa por dos puntos conocidos y que permite determinar la ordenada de otro punto de esa recta a partir de su abscisa. Esta función es una de las muchas ecuaciones relacionadas con geometría que se le proporciona al alumno. Nosotros evaluamos que el alumno haya comprendido que por la física del proceso existe una línea que conecta esos puntos y no que el alumno sepa la expresión matemática de la recta que pasa por dos puntos. Del mismo modo, como ya se ha comentado, la explicación del proceso se basa en construcciones gráficas, por ello, tras llegar a la solución numérica,

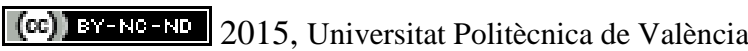


lo importante para el aprendizaje del alumno es que se centre en comprobar la solución e interpretarla, relacionándola con lo aprendido en la teoría, y no que se pierda en la elaboración del gráfico a partir de las sentencias propias del software utilizado. La Figura 4 muestra uno de los gráficos que se proporcionan junto al enunciado del ejercicio.

Las funciones construidas que se proporcionan a los alumnos para su aplicación son:

- Cálculos muy sencillos y repetidos relacionados con construcciones geométricas: líneas, intersecciones, tangencias, etc.

- Funciones de interpolación para el tratamiento de datos termodinámicos.

- Subrutinas para cálculos estándar: reparto.

- Gráficos construidos a partir de los resultados obtenidos.

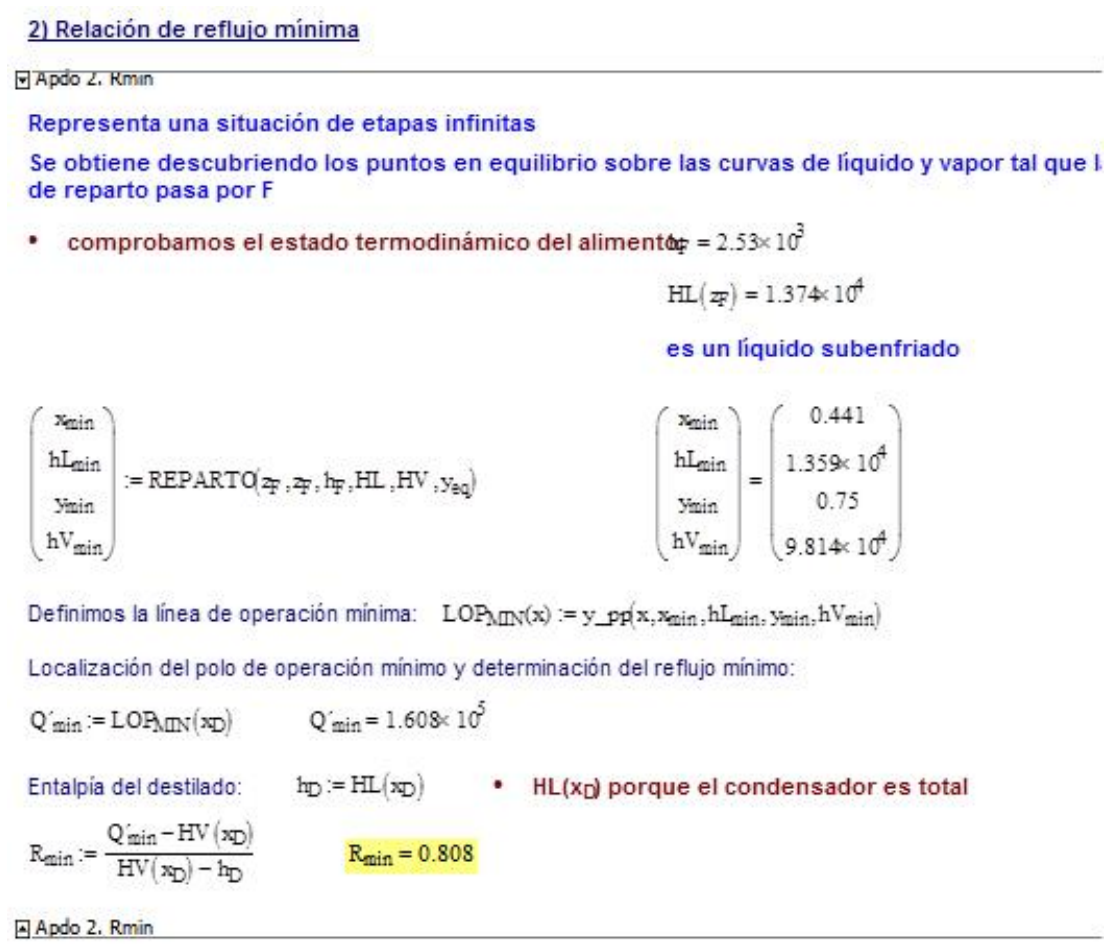

Fig. 3. Aplicación de una función de cálculo creada a la resolución de un cálculo. 


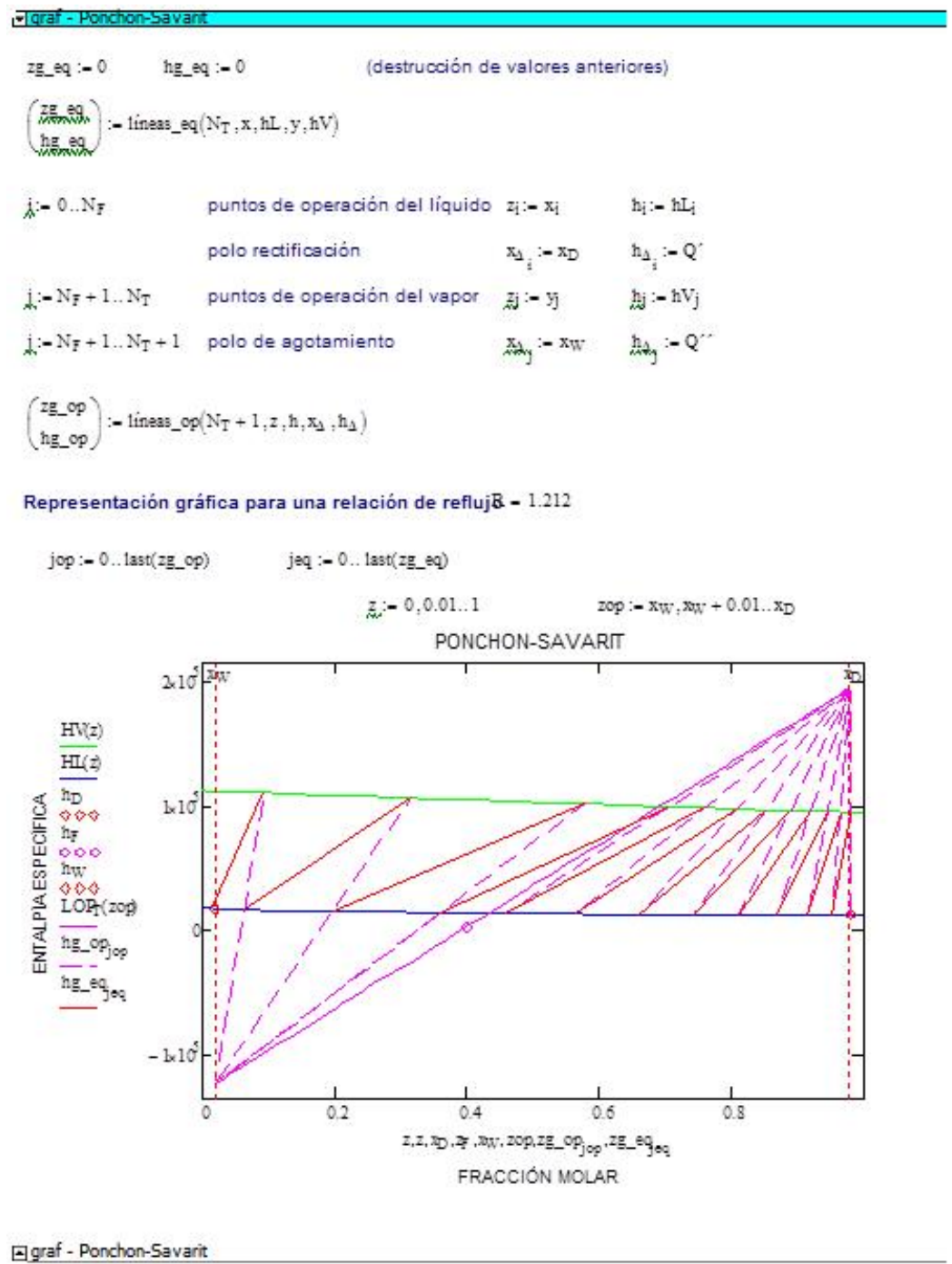

Fig. 4. Aplicación de un método gráfico construido para analizar los resultados obtenidos.

Como consecuencia del análisis anterior se reestructuraron los problemas a partir de las funcionalidades del software. Se aprovechó la creación de "áreas” en la hoja de cálculo para delimitar zonas en las que realizar cálculos concretos que se pueden plegar una vez realizados y permiten crear una especie de índice de los cálculos que hace que el seguimiento del proceso de cálculo sea más visual. Como se ha ilustrado arriba con la función "reparto", también se aprovechó está funcionalidad para la creación de librerías de funciones de cálculos que, como se ha comentado, se proporcionan directamente a los

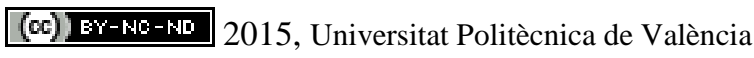

Congreso In-Red (2015) 
alumnos. La Figura 5 muestra el cálculo completo de un ejercicio. Las zonas coloreadas son las funciones ya definidas que se proporcionan junto con el enunciado del ejercicio. Como se puede observar, una de estas funciones es la del cálculo del reparto y la del gráfico de la solución alcanzada; el resto son las zonas donde los alumnos realizan sus cálculos, entre los que se incluye el cálculo mostrado en la Figura 3. De esta manera, se puede observar que la definición matemática y la resolución de la función reparto es sólo un paso dentro de los muchos que el alumno debe realizar para completar el ejercicio. Se puede comprobar que, con la creación de la función, se evita que el alumno pierda tiempo en la definición matemática de un cálculo geométrico y no desvíe su atención de los cálculos propios de la operación de separación estudiada.

Con la estructuración de los problemas, resultó más fácil para los profesores transmitir a los alumnos, la similitud de los cálculos requeridos en las distintas operaciones y cómo los cálculos desarrollados en una operación son extrapolables a los cálculos de las otras.

A partir de este esquema más visual de los pasos seguidos para la resolución de los cálculos de la operación de la Figura 5 es fácil establecer una comparación con los pasos realizados en operaciones previamente practicadas en aula informática y establecer las relaciones y similitudes.

El empleo de zonas de trabajo también hizo más fácil el planteamiento del examen y sobretodo su corrección, cosa importante teniendo en cuenta el elevado número de alumnos matriculados. 


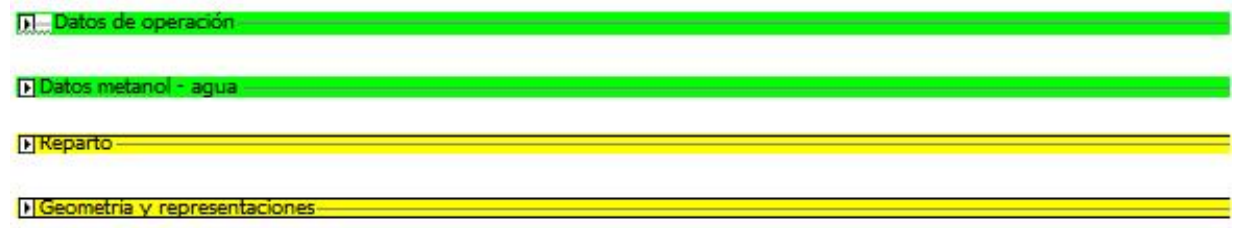

1. Cantidad de productos

冈- Apdo 1. D y W

\section{Relación de reflujo mínima}

[. Apdo 2. Rmin

\section{Carqas térmicas del condensador y calderín}

1.-Apdo 3. Cargas térmicas

\section{Cálculo de las etapas ideales de la rectificación}

1.- Apdo 4. Polos de operación y punto de cambio

口Apdo 4. Etapas

d. graf-ponchon-5avant

\section{$\underline{\text { 5. Perfiles de fluio, composición y temperatura }}$}

17. Apdo 5. Perfiles en la torre

\section{Darat - Periles en la torn}

6. Número mínimo de etapas

G Apdo 6. Etapas mínimas

Đgraf - PS Nmin

Fig. 5. Esquema del proceso de cálculo realizado para calcular un proceso de rectificación mediante el método de Ponchon-Savarit.

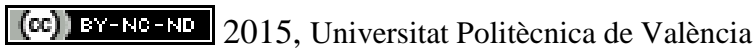

Congreso In-Red (2015) 
Análisis estructural de los problemas orientado al aprendizaje de Operaciones de Separación

\section{Conclusiones}

La adaptación de los problemas ha aportado los siguientes beneficios:

- El uso de zonas de trabajo permite que los ejercicios sean más compactos y visuales, quedando identificada de forma más clara la estructura del ejercicio. Con ello, el alumno tiene menos probabilidades de perderse dentro de un gran conjunto de ecuaciones y puede centrar su atención en la resolución de pasos específicos.

- La creación de una librería de funciones ha permitido que el tiempo de realización de un problema sea más corto, adaptándose a la duración de una clase de problemas de dos horas.

- Se facilita la comprensión de problemas más complejos y que no siguen la secuencia tradicional de cálculo. El alumno que comprende cuales son los elementos del primer nivel de subdivisión de un problema debería ser capaz de razonar otras secuencias de cálculo diferentes.

- El alumno que comprenda la similitud entre las operaciones debe ser capaz de extrapolar y adaptar un cálculo concreto que se desarrolla en clase en una operación al cálculo de otra.

- Los resultados de aprendizaje expuestos en los ejemplos arriba indicados son fácilmente detectables en un ejercicio de examen.

- En lo que respecta a la evaluación, la estructuración ha permitido facilitar la corrección pues el alumno realiza la resolución de forma más ordenada.

En este primer año de introducción de la metodología, los logros obtenidos en cuanto a la percepción de los alumnos se han evaluado mediante una encuesta que mostró los siguientes resultados:

- El 80\% de los alumnos piensa que "La división en apartados de los problemas me ha ayudado a comprender la estructura de los cálculos y los pasos a realizar para llegar a la solución final” en un grado “Alto” y "Muy alto”.

- Sin embargo, el porcentaje en ese grado desciende a un 62\% cuando la pregunta realizada es “¿En qué medida has identificado que los problemas realizados en diferentes operaciones presentaban ciertas similitudes en su estructura (secuencia de pasos) de resolución?”. Esto evidencia que habrá que seguir haciendo más hincapié en este aspecto.

- El 73\% de los alumnos responde con un grado “Alto” y “Muy alto” a la pregunta “¿En qué medida sabrías extrapolar a la resolución de problemas de otras asignaturas la forma estructurada de resolución de un problema tal y como se ha practicado en operaciones de separación?”

A este respecto, para seguir evaluando los logros conseguidos es necesario añadir más herramientas de medición. Así, el próximo paso será incluir preguntas de razonamiento en

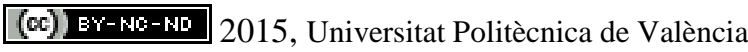

Congreso IN-RED (2015) 
los exámenes y de extrapolación de situaciones entre operaciones, como forma más objetiva de evaluar el grado de integración alcanzado por los alumnos. Del mismo modo, se irá analizando el progreso de la innovación comparando las notas obtenidas por los alumnos desde que se ha comenzado a impartir la asignatura del Grado.

Los autores pensamos que si bien la metodología descrita se ha aplicado a una materia concreta (Operaciones de Separación), el enfoque del trabajo es aplicable en otras asignaturas.

\section{Agradecimientos}

Los miembros del grupo ASEI agradecen al Vicerrectorado de Estudios y Convergencia Europea y al Instituto de Ciencias de Educación de la Universitat Politècnica de València la concesión del PIME A26/14 "Desarrollo de estrategias para la mejora del aprendizaje y evaluación de los problemas de Operaciones de Separación”

\section{Bibliografía}

Santafé Moros, A., Gozálvez Zafrilla, J.M., Lora García, J. (2013). Cálculo de operaciones de separación con Mathcad ${ }^{\circledR}$. Valencia: Editorial UPV.

HARB, J.N., JONES, A., ROWLEY, R.L., WILDING, W.V. (1997). "Use of computational tools in engineering education. A case study on the use of Mathcad®" en Chemical Engineering Education, Summer 1997, pp. 180-187.

SÁNChez Mirón, A., Cerón García, M. C., GarCía Camacho, F., Esteban Cerdán, L., GonzÁLez Moreno, P. A. Mathcad como herramienta de aprendizaje en la ingeniería química. En I Congreso de innovación docente en Ingeniería Química. Granada. Disponible en <http://www.coddiq.es/cidiq2012/images/stories/comunicaciones/1232o2.pdf> [Consulta: 20 de mayo de 2015] 